\title{
Variation in Phenotypic Characteristics of Phytophthora capsici Isolates from a Worldwide Collection
}

L. L. Granke, Visiting Research Associate, L. M. Quesada-Ocampo, Visiting Research Associate, and M. K. Hausbeck, Professor, Department of Plant Pathology, Michigan State University, East Lansing 48824

\begin{abstract}
Granke, L. L., Quesada-Ocampo, L. M., and Hausbeck, M. K. 2011. Variation in phenotypic characteristics of Phytophthora capsici isolates from a worldwide collection. Plant Dis. 95:1080-1088.

To determine variation within Phytophthora capsici, 124 P. capsici isolates from 12 countries were characterized for sporangial length and width, pedicle length, oospore diameter, sporangia and chlamydospore production, and growth at 32,35 , and $38^{\circ} \mathrm{C}$. Sporangia were 23 to 35 $\mu \mathrm{m}$ wide and 38 to $60 \mu \mathrm{m}$ long; differences in width and length were noted when isolates were grouped by genetic cluster and continent of origin. Length:breadth ratio (1.34 to 2.07) and pedicle length (20 to $260 \mu \mathrm{m}$ long) varied widely among isolates; differences were apparent by continent and host family of origin. Oospore diameters varied

genetic cluster, host family of origin, continent of origin, mating type, or sensitivity to mefenoxam. Differences in sporangia production were observed among isolates grouped by continent, and isolates from nonvegetable hosts produced fewer sporangia than isolates from vegetable hosts. When cultures were incubated in a liquid medium, 35 P. capsici isolates formed chlamydospores. Most (122 of 124) of the isolates were able to grow at $35^{\circ} \mathrm{C}$, but all of the isolates grew poorly at $38^{\circ} \mathrm{C}$. The results of this study indicate substantial variation in morphological and physiological characteristics among $P$. capsici isolates.
\end{abstract} among isolates ( 22 to $37 \mu \mathrm{m}$ ), but no differences were noted by isolate
The oomycete plant pathogen Phytophthora capsici Leonian is a serious pathogen that limits production of vegetable crops in the Cucurbitaceae, Solanaceae, and Fabaceae (16). The pathogen is distributed worldwide (9) and may also cause disease on nonvegetable hosts such as cacao (Theobroma cacao L.), rubber (Hevea brasiliensis Mull. Arg.), macadamia (Macadamia integrifolia Maiden \& Betche), papaya (Carica papaya L.), and black pepper (Piper nigrum L.) (9). P. capsici is heterothallic and requires both the A1 and A2 mating types (MTs) to be present for oospore production (19). Challenges to managing disease caused by $P$. capsici include long-term survival of oospores in the soil (20), an increasing list of susceptible hosts (25), fungicide-resistant pathogen populations (18), spread of the pathogen via irrigation water (13), and a lack of commercially acceptable resistant cultivars $(11,12,21)$.

Identifying the causal agent of Phytophthora root, stem, and fruit rot may be challenging, as many of the hosts susceptible to $P$. capsici are susceptible to other Phytophthora species and $P$. capsici displays considerable phenotypic variation (9). This is especially true for nonvegetable hosts such as cacao, which may be infected with $P$. capsici, P. palmivora, P. megakarya, P. citrophthora, $P$. nicotianae (2), and $P$. tropicalis (8). However, diseases on vegetable crops may also be caused by species other than $P$. capsici; root rot of tomato is caused by $P$. capsici and $P$. nicotianae $(15,27)$. Nonetheless, correct identification of the causal agent is important for disease management and also for the correct use of pathogen isolates in resistance screening programs. Previous studies have used morphology (3), species-specific polymerase chain reaction (PCR) (30), PCR and sequencing (4,26), and enzyme digestion $(4,22)$ to separate $P$. capsici from closely related species.

Differences in morphological and physiological characters of $P$. capsici isolates have been previously reported $(3,4,17,22,28,31)$. Sporangial lengths have been previously reported to range from 33

Corresponding author: M. K. Hausbeck, E-mail: hausbec1@msu.edu

Accepted for publication 9 May 2011.

doi:10.1094/PDIS-03-11-0190

(c) 2011 The American Phytopathological Society to $66 \mu \mathrm{m}$ and widths from 17 to $43 \mu \mathrm{m}(3,17,22,28)$. Overall, the length:breadth (LB) ratio of sporangia ranged from $<1.3$ to 2.2 in prior work $(3,9,22,28)$. Pedicle lengths for $P$. capsici isolates are known to vary widely; pedicles for isolates from a variety of hosts ranged from 32 to $138 \mu \mathrm{m}$ long $(17,22,28)$. Chlamydospores formed in liquid cultures of some isolates from nonvegetable hosts $(4,22)$ and pumpkin (Cucurbita moschata Duchesne ex Poir.) (17), but were not observed for isolates from pepper (Capsicum annuum L.), tomato (Solanum lycopersicum L.), eggplant (Solanum melongena L.), lima bean (Phaseolus lunatus L.), cucumber (Cucumis sativus L.), or squash (Cucurbita pepo L.) $(4,28)$. Because of the variability in morphological characteristics among isolates, identifying Phytophthora species based on morphology alone may be challenging.

Much genetic variability exists within $P$. capsici (10), and collections of isolates have been separated into subpopulations using isozymes (22,23), traditional phylogenetic methods $(4,8)$, and Bayesian clustering (26). When a group of 114 isolates from vegetable and nonvegetable hosts were separated into two subgroups (CAPA and CAPB) using isozymes, similarities in sporangial morphology were noted within each group (22). However, a few isolates from each subgroup showed sporangial morphology characteristic of the opposite group. Only isolates in CAPB $(P$. tropicalis) produced chlamydospores (22); whereas CAPA ( $P$. capsici sensu stricto) isolates did not produce chlamydospores in this study (22). Other studies have correlated genetic subgroups with host of origin and geography $(4,8,23)$, but did not investigate correspondence with morphological characteristics. When Bayesian clustering was used to subdivide a worldwide collection of $P$. capsici isolates (26), no direct correlation was observed between genetic clusters and host of origin, geography, or mefenoxam sensitivity, but population structure was apparent when isolates were grouped by these categories. Previous studies have not investigated if morphological and physiological differences correspond with genetic clusters or subpopulations. Since characterizing population subdivision with molecular tools can be a time-consuming and costly procedure, it would be helpful if phenotypic characteristics could be used to guide the sampling process.

There are conflicting opinions as to whether or not $P$. tropicalis should be considered a separate species from $P$. capsici $(3,4)$. While morphological (3) and genetic $(8,35)$ differences may be 
observed among $P$. capsici and $P$. tropicalis, overlap may also be observed between some isolates of these species. Previous studies $(3,4,22)$ have suggested that growth at $35^{\circ} \mathrm{C}$ may be used to separate $P$. capsici from $P$. tropicalis. Identification of Phytophthora species using morphological characteristics is challenging in general (5), and species like $P$. capsici may have much intra-specific variation in morphological characteristics $(9,28)$. Even when molecular methods are used, isolates may be misidentified unless information from multiple genes is used in the analysis. QuesadaOcampo et al. (26) found isolates belonging to P. capsici, P. tropicalis, and others with an intermediate genotype within a set of 255 $P$. capsici sensu lato isolates. While $P$. capsici and $P$. tropicalis isolates were assigned to their respective species for every gene, the intermediate isolates were assigned to $P$. capsici for some genes and to $P$. tropicalis for others. These intermediate isolates would have been incorrectly identified as $P$. capsici or $P$. tropicalis if only a single gene was used. Hence, investigating the intraspecific diversity of phenotypic characteristics of a large set of $P$. capsici isolates from diverse hosts and geographic locations may aid understanding the natural phenotypic variation of $P$. capsici and the overlap between $P$. capsici and $P$. tropicalis, which could help guide separation of these closely related species.

The objective of this study was to determine variations in sporangial morphology, sporangial and chlamydospore production, and growth at 32,35 , and $38^{\circ} \mathrm{C}$ for $P$. capsici populations from diverse hosts and geographic locations. Isolates were grouped within arbitrarily defined geographic, host, mefenoxam resistance, and mating type categories or by predominant genetic cluster membership, and differences between subgroups in each category were investigated. A small set of $P$. tropicalis isolates and isolates with an intermediate genotype between $P$. capsici and $P$. tropicalis were included for comparison. A preliminary report of morphological characters for some of the isolates included in this study has been published (14), and the species identity, mating type, mefenoxam sensitivity, and genetic cluster membership of isolates included in this study have been previously reported (26).

\section{Materials and Methods}

Isolate selection, maintenance, and genetic cluster assignment. A total of 124 P. capsici isolates were chosen from the culture collection maintained in the laboratory of Dr. Hausbeck at Michigan State University (MSU). Isolates from 12 countries and 17 host species were collected or were donated by colleagues in various countries (Table 1). Single-spore cultures were retrieved from long-term storage and maintained on unclarified V8 agar (UCV8, $3 \mathrm{~g} \mathrm{CaCO}_{3}, 15 \mathrm{~g}$ agar, $160 \mathrm{ml}$ unfiltered V8 juice, and 840 $\mathrm{ml}$ distilled water) as previously described (25). P. capsici was confirmed using morphological characteristics according to the Phytophthora spp. key by Waterhouse (33) and molecular techniques (26). We included the type cultures for $P$. capsici 13692 (ATCC.15399, New Mexico) and P. tropicalis 13602 (ATCC.76651, Hawaii) and additional $P$. tropicalis and intermediate isolates for a total of 138 isolates (124 P. capsici, 9 P. tropicalis, and 5 isolates with an intermediate genotype).

The model-based Bayesian clustering algorithm implemented in Structure 2.3X (24) was used in a previous study to assess population subdivision for a group of 236 P. capsici sensu stricto isolates, 124 of which are included in this study (26). Each isolate was assigned proportionate membership (Q) in a genetic cluster by Structure 2.3X. For statistical analysis, each isolate was assigned to the cluster in which it had predominant membership. If an isolate's membership was not $>0.1$ proportion membership higher in one genetic cluster than another, that isolate was designated as highly admixed and excluded from statistical analysis $(n=9)$, but not from population structure figures. The graphic editing program Population Sorting Tool (PST) (J. J. Morrice, unpublished data) was used to create population structure figures for $P$. capsici with phenotypic information and isolates sorted by Q.

Phenotypic characterization. The length, breadth, and pedicel length of 10 arbitrarily chosen sporangia were measured for each isolate. To dislodge sporangia, a droplet of water was added to each single culture, agitated, and poured onto a glass slide. An optical micrometer and compound microscope were used to measure sporangial length and breadth at $\times 200$ magnification and pedicle length at $\times 100$ magnification. The length:breadth (LB) ratio was calculated for each isolate. The experiment was conducted twice.

Sporangial production on culture medium was estimated for each isolate. To estimate sporangial production, a 6-mm-diameter mycelial disk was transferred from the growing edge of a 7-dayold culture onto three replicate 60 -mm-diameter plastic petri dishes containing UCV8. Plates were incubated in a single layer under constant fluorescent lighting at room temperature $\left(25 \pm 2^{\circ} \mathrm{C}\right)$ for 7 days. For each plate, $1 \mathrm{ml}$ of sterile distilled water was added to the culture, sporangia were dislodged using a sterilized bacterial loop, and the resulting suspension was pipetted into a $1.7-\mathrm{ml}$ microcentrifuge tube. Water was added to the tube as needed to ensure that $1 \mathrm{ml}$ of suspension was present in each tube. For each replicate of each isolate, sporangia were enumerated using a hemacytometer, and the number of sporangia per square centimeter of medium was calculated. The experiment was conducted twice.

Since the mating type of each isolate had been previously determined (26), isolates were paired with a known standard of the opposite mating type (either isolate 101 or 13349) on UCV8 to form oospores. The diameters of 10 oospores per isolate were measured using an optical micrometer at $\times 200$ magnification by taking a measurement of the length and width at the widest point (excluding the antheridium).

To determine if isolates would produce chlamydospores in liquid medium, the methods of Ristaino (28) were followed with modifications. Briefly, a single 8-mm-diameter plug from the growing edge of each isolate was transferred to $25 \mathrm{ml}$ of clarified V8 broth (C-V8, $163 \mathrm{ml}$ clarified V8 juice, $3 \mathrm{~g} \mathrm{CaCO}_{3}, 1$ liter distilled water, $86 \mathrm{mg}$ ampicillin, $26 \mathrm{mg}$ rifampicin) in a 50-ml Corning tube and incubated vertically in the dark at room temperature $\left(\sim 25 \pm 2^{\circ} \mathrm{C}\right)$ for $24 \mathrm{~h}$. The tubes were shaken vigorously for $5 \mathrm{~min}$ using a shaker and then incubated vertically for 5 days in the dark at room temperature. The $\mathrm{C}-\mathrm{V} 8$ broth was removed from the tube and was replaced with $\sim 45 \mathrm{ml}$ of sterile distilled water. Cultures were incubated vertically in an incubator at $18 \pm 2^{\circ} \mathrm{C}$ for 3 weeks with no light and checked for the presence of chlamydospores. Each culture was individually removed from the tube in which it was incubated and placed into a plastic $80-\mathrm{mm}$-diameter petri dish. The mycelia were teased apart using forceps and a dissecting needle to enable visualization, and the culture was scanned under $\times 100$ magnification for chlamydospores.

To examine isolate growth at temperatures near the maximum growth temperature (9), a 6-mm-diameter agar plug from the growing edge of a 5-day-old culture of each isolate was inoculated to the center of six $60-\mathrm{mm}$-diameter UCV8 plates. Two replicate plates per isolate were Parafilmed and incubated in the dark in growth chambers set at 32,35 , and $38^{\circ} \mathrm{C}$, respectively. The temperature in each growth chamber was monitored for the duration of each experiment using a WatchDog model 450 data logger (Spectrum Technologies, Plainfield, IL). Data loggers were set to record temperature $\left({ }^{\circ} \mathrm{C}\right)$ at 10 -min intervals. The accuracy ratings of the data loggers were $\pm 0.6^{\circ} \mathrm{C}$. Data were downloaded into a computer at the end of each experiment. The diameter of pathogen growth was measured after 5 days incubation and recorded. The experiment was conducted twice. The recorded temperature in each growth chamber was $32.2 \pm 0.2,35.3 \pm 0.0$, and $38.3 \pm 0.1^{\circ} \mathrm{C}$ for the first experiment and $32.8 \pm 0.1,35.7 \pm 0.2$, and $38.7 \pm 0.2^{\circ} \mathrm{C}$ for the second experiment, respectively.

Data analysis. All statistical analyses were performed using the SAS statistical package version 9.1 (SAS Institute, Cary, NC). Isolates were grouped by species and subjected to analysis of variance. Tukey's HSD was used for separation of means to account for unequal sample sizes between subgroups in a category $(P=$ 0.05). Isolate was considered a random factor in the ANOVA analyses. The response variables were sporangial width, sporangial 
Table 1. Isolates of Phytophthora species used in this study

\begin{tabular}{|c|c|c|c|c|c|c|c|}
\hline \multirow{2}{*}{$\frac{\text { Isolate }}{101}$} & \multicolumn{2}{|c|}{ Origin $^{v}$} & \multirow{2}{*}{$\frac{\text { Host family }}{\text { Cucurbitaceae }}$} & \multirow{2}{*}{$\frac{\mathbf{M T}^{\mathbf{w}}}{\mathrm{A} 1}$} & \multirow{2}{*}{$\frac{\mathbf{M S}^{\mathbf{x}}}{\mathrm{S}}$} & \multirow{2}{*}{$\frac{\mathbf{G C}^{\mathbf{y}}}{7}$} & \multirow{2}{*}{$\frac{\text { Source }^{\mathbf{z}}}{\text { M. K. Hausbeck }}$} \\
\hline & N. America & US, MI & & & & & \\
\hline 102 & N. America & US, MI & Cucurbitaceae & A2 & I & 3 & M. K. Hausbeck \\
\hline 455 & N. America & US, MI & Cucurbitaceae & $\mathrm{A} 1$ & $\mathrm{~S}$ & 3 & M. K. Hausbeck \\
\hline 1177 & N. America & US, MI & Cucurbitaceae & A2 & $\mathrm{S}$ & 3 & M. K. Hausbeck \\
\hline 9790 & N. America & US, MI & Solanaceae & $\mathrm{A} 1$ & IS & $\mathrm{AD}$ & M. K. Hausbeck \\
\hline 9964 & N. America & US, MI & Cucurbitaceae & A1 & $\mathrm{S}$ & 3 & M. K. Hausbeck \\
\hline 10044 & N. America & US, MI & Solanaceae & A2 & $\mathrm{S}$ & 3 & M. K. Hausbeck \\
\hline 10193 & N. America & US, MI & Fabaceae & $\mathrm{A} 1$ & $\mathrm{~S}$ & 5 & M. K. Hausbeck \\
\hline 10213 & N. America & US, MI & Fabaceae & A1 & I & 5 & M. K. Hausbeck \\
\hline 10251 & N. America & US, MI & Fabaceae & $\mathrm{A} 1$ & $\mathrm{~S}$ & 5 & M. K. Hausbeck \\
\hline 10412 & N. America & US, MI & Cucurbitaceae & A2 & I & 3 & M. K. Hausbeck \\
\hline 10858 & N. America & US, MI & Fabaceae & $\mathrm{A} 1$ & $\mathrm{~S}$ & 5 & M. K. Hausbeck \\
\hline 11457 & N. America & US, MI & Solanaceae & A1 & IS & 3 & M. K. Hausbeck \\
\hline 11469 & N. America & US, MI & Solanaceae & $\mathrm{A} 2$ & IS & 5 & M. K. Hausbeck \\
\hline 11478 & N. America & US, MI & Fabaceae & A1 & I & 5 & M. K. Hausbeck \\
\hline 11571 & N. America & US, MI & Cucurbitaceae & A2 & $\mathrm{S}$ & 3 & M. K. Hausbeck \\
\hline 11783 & N. America & US, MI & Cucurbitaceae & A2 & I & 3 & M. K. Hausbeck \\
\hline 11861 & N. America & US, MI & Cucurbitaceae & A2 & IS & 5 & M. K. Hausbeck \\
\hline 11885 & N. America & US, MI & Cucurbitaceae & A1 & I & 5 & M. K. Hausbeck \\
\hline 11923 & N. America & US, MI & Cucurbitaceae & A1 & I & 5 & M. K. Hausbeck \\
\hline 12017 & N. America & US, MI & Cucurbitaceae & A2 & S & 3 & M. K. Hausbeck \\
\hline 12162 & N. America & US, MI & Cucurbitaceae & A2 & $\mathrm{S}$ & 3 & M. K. Hausbeck \\
\hline 12328 & N. America & US, MI & Solanaceae & A1 & IS & 3 & M. K. Hausbeck \\
\hline 12842 & N. America & US, MI & Solanaceae & A2 & $\mathrm{S}$ & 3 & M. K. Hausbeck \\
\hline 12889 & N. America & US, MI & Solanaceae & A1 & I & 5 & M. K. Hausbeck \\
\hline 13199 & N. America & US, KY & Cucurbitaceae & A1 & $\mathrm{S}$ & 4 & K. W. Seebold \\
\hline 13200 & N. America & US, KY & Cucurbitaceae & A2 & $\mathrm{S}$ & 8 & K. W. Seebold \\
\hline 13201 & N. America & US, KY & Cucurbitaceae & A2 & $\mathrm{S}$ & 8 & K. W. Seebold \\
\hline 13202 & N. America & US, NY & Cucurbitaceae & A2 & $\mathrm{S}$ & 5 & C. D. Smart \\
\hline 13204 & N. America & US, NY & Solanaceae & A1 & $\mathrm{S}$ & $\mathrm{AD}$ & C. D. Smart \\
\hline 13205 & N. America & US, NY & Cucurbitaceae & A2 & $\mathrm{S}$ & 4 & C. D. Smart \\
\hline 13206 & N. America & US, NY & Cucurbitaceae & A1 & $\mathrm{S}$ & 5 & C. D. Smart \\
\hline 13207 & N. America & US, CA & Solanaceae & $\mathrm{A} 2$ & S & 9 & C. L. Blomquist \\
\hline 13208 & N. America & US, CA & Solanaceae & A2 & $\mathrm{S}$ & 9 & C. L. Blomquist \\
\hline 13212 & N. America & US, SC & Cucurbitaceae & A2 & $\mathrm{S}$ & 8 & A. P. Keinath \\
\hline 13213 & N. America & US, SC & Cucurbitaceae & A2 & $\mathrm{S}$ & 8 & A. P. Keinath \\
\hline 13214 & N. America & US, SC & Cucurbitaceae & A2 & $\mathrm{S}$ & 8 & A. P. Keinath \\
\hline 13219 & N. America & US, SC & Cucurbitaceae & A1 & S & 4 & A. P. Keinath \\
\hline 13220 & N. America & US, SC & Cucurbitaceae & A1 & $\mathrm{S}$ & 4 & A. P. Keinath \\
\hline 13222 & N. America & US, SC & Solanaceae & A1 & S & 2 & A. P. Keinath \\
\hline 13224 & N. America & US, SC & Solanaceae & A2 & $\mathrm{S}$ & $\mathrm{AD}$ & A. P. Keinath \\
\hline 13226 & N. America & US, SC & Solanaceae & A2 & I & 4 & A. P. Keinath \\
\hline 13228 & N. America & US, SC & Solanaceae & $\mathrm{A} 1$ & I & 4 & A. P. Keinath \\
\hline 13229 & N. America & US, SC & Solanaceae & A1 & IS & 5 & A. P. Keinath \\
\hline 13230 & N. America & US, SC & Solanaceae & A1 & $\mathrm{S}$ & 4 & A. P. Keinath \\
\hline 13233 & N. America & US, LA & Solanaceae & A1 & $\mathrm{S}$ & $\mathrm{AD}$ & D. M. Ferrin \\
\hline 13236 & N. America & US, AR & Solanaceae & A2 & $\mathrm{S}$ & 4 & M. E. Matheron \\
\hline 13239 & N. America & US, TX & Cucurbitaceae & A2 & $\mathrm{S}$ & 9 & T. Isakeit \\
\hline 13243 & N. America & US, NC & Cucurbitaceae & A1 & S & 5 & K. L. Ivors \\
\hline 13245 & N. America & US, NC & Cucurbitaceae & A1 & $\mathrm{S}$ & 5 & K. L. Ivors \\
\hline 13247 & N. America & US, NC & Cucurbitaceae & A1 & $\mathrm{S}$ & 4 & K. L. Ivors \\
\hline 13248 & N. America & US, NC & Cucurbitaceae & A1 & S & 5 & K. L. Ivors \\
\hline 13249 & N. America & US, NC & Cucurbitaceae & A1 & $\mathrm{S}$ & 5 & K. L. Ivors \\
\hline 13338 & N. America & US, MA & Cucurbitaceae & A2 & S & 3 & R. L. Wick \\
\hline 13339 & N. America & US, MA & Cucurbitaceae & $\mathrm{A} 1$ & $S$ & 3 & R. L. Wick \\
\hline 13347 & N. America & US, GA & Cucurbitaceae & A1 & $\mathrm{S}$ & 8 & P. Ji \\
\hline 13349 & N. America & US, MI & Cucurbitaceae & $\mathrm{A} 2$ & $\mathrm{~S}$ & 7 & M. K. Hausbeck \\
\hline 13366 & S. America & Brazil & Sterculiaceae & A1 & $\mathrm{S}$ & IN & K. H. Lamour \\
\hline 13367 & S. America & Brazil & Sterculiaceae & $\mathrm{A} 2$ & S & IN & K. H. Lamour \\
\hline 13368 & S. America & Brazil & Sterculiaceae & A2 & $\mathrm{S}$ & IN & K. H. Lamour \\
\hline 13371 & Africa & Cameroon & Sterculiaceae & A1 & S & 1 & K. H. Lamour \\
\hline 13377 & N. America & Mexico & Sterculiaceae & $\mathrm{A} 1$ & $S$ & TR & K. H. Lamour \\
\hline 13384 & Asia & India & Piperaceae & A2 & $\mathrm{S}$ & TR & K. H. Lamour \\
\hline 13385 & N. America & US, HI & Proteaceae & A2 & $\mathrm{S}$ & TR & K. H. Lamour \\
\hline 13390 & N. America & Mexico & Solanaceae & A2 & $\mathrm{S}$ & 4 & K. H. Lamour \\
\hline 13397 & N. America & US, HI & Proteaceae & $\mathrm{A} 2$ & $\mathrm{~S}$ & 6 & K. H. Lamour \\
\hline & & & & & & & (continued on next page) \\
\hline
\end{tabular}

${ }^{\mathrm{v}}$ Geographical origin (continent, country, state). Standard U.S. state abbreviations are used.

${ }^{\text {w }}$ Mating type of an isolate: A1 or A2.

${ }^{x}$ Sensitivity to mefenoxam: $\mathrm{I}=$ insensitive, IS = intermediately insensitive, $\mathrm{S}=$ sensitive.

${ }^{y}$ Predominant genetic cluster (GC) membership. AD indicates a highly admixed $P$. capsici isolate. TR indicates $P$. tropicalis. IN indicates isolates with an intermediate genotype.

z ATCC = American Type Culture Collection 
Table 1. (continued from previous page)

\begin{tabular}{|c|c|c|c|c|c|c|c|}
\hline \multirow{2}{*}{$\frac{\text { Isolate }}{13398}$} & \multicolumn{2}{|c|}{ Origin $^{v}$} & \multirow{2}{*}{$\begin{array}{l}\text { Host family } \\
\text { Sterculiaceae }\end{array}$} & \multirow{2}{*}{$\frac{\mathbf{M T}^{\mathbf{w}}}{\mathrm{A} 2}$} & \multirow{2}{*}{$\frac{\text { MS }^{\mathbf{x}}}{S}$} & \multirow{2}{*}{$\frac{\mathbf{G C}^{\mathbf{y}}}{\mathrm{TR}}$} & \multirow{2}{*}{$\frac{\text { Source }^{\mathbf{z}}}{\text { K. H. Lamour }}$} \\
\hline & N. America & Mexico & & & & & \\
\hline 13401 & N. America & US, CA & Cucurbitaceae & A2 & $\mathrm{S}$ & 8 & K. H. Lamour \\
\hline 13407 & Europe & Norway & Cucurbitaceae & $\mathrm{A} 2$ & $\mathrm{~S}$ & 8 & M. L. Herrero \\
\hline 13423 & N. America & US, MI & Cucurbitaceae & $\mathrm{A} 1$ & $\mathrm{~S}$ & 3 & M. K. Hausbeck \\
\hline 13456 & N. America & US, MI & Cucurbitaceae & A1 & $\mathrm{S}$ & 3 & M. K. Hausbeck \\
\hline 13471 & N. America & US, MI & Solanaceae & A2 & I & 3 & M. K. Hausbeck \\
\hline 13479 & Europe & Spain & Solanaceae & A1 & $\mathrm{S}$ & 8 & A. Lacasa \\
\hline 13602 & N. America & US, HI & Proteaceae & $\mathrm{A} 1$ & $\mathrm{~S}$ & TR & ATCC \\
\hline 13603 & N. America & US, HI & Caricaceae & A2 & $\mathrm{S}$ & TR & J. Y. Uchida \\
\hline 13606 & Australia & Australia & Annonaceae & $\mathrm{A} 1$ & $\mathrm{~S}$ & IN & B. McNeil \\
\hline 13607 & Australia & Australia & Apocynaceae & $\mathrm{A} 1$ & $\mathrm{~S}$ & TR & B. McNeil \\
\hline 13608 & Australia & Australia & Annonaceae & A2 & S & TR & B. McNeil \\
\hline 13609 & Australia & Australia & Annonaceae & $\mathrm{A} 2$ & $\mathrm{~S}$ & TR & B. McNeil \\
\hline 13613 & N. America & US, DE & Fabaceae & A1 & I & 7 & G. Majeau \\
\hline 13614 & N. America & US, DE & Fabaceae & $\mathrm{A} 1$ & $\mathrm{~S}$ & IN & G. Majeau \\
\hline 13616 & N. America & US, FL & Solanaceae & A1 & $\mathrm{S}$ & 5 & A. J. Gevens \\
\hline 13617 & N. America & US, FL & Solanaceae & $\mathrm{A} 1$ & $\mathrm{~S}$ & 5 & A. J. Gevens \\
\hline 13620 & Europe & Italy & Solanaceae & $\mathrm{A} 1$ & $\mathrm{~S}$ & 6 & R. B. Kung \\
\hline 13621 & Europe & Italy & Solanaceae & A2 & $\mathrm{S}$ & 6 & R. B. Kung \\
\hline 13624 & Europe & Italy & Solanaceae & $\mathrm{A} 1$ & $\mathrm{~S}$ & 6 & G. Tamietti \\
\hline 13632 & Europe & Italy & Solanaceae & A1 & $\mathrm{S}$ & 6 & G. Tamietti \\
\hline 13634 & Europe & Italy & Solanaceae & $\mathrm{A} 1$ & $\mathrm{~S}$ & 9 & G. Tamietti \\
\hline 13638 & Asia & Taiwan & Solanaceae & A2 & $\mathrm{S}$ & 3 & T. C. Wang \\
\hline 13639 & Asia & Taiwan & Solanaceae & A1 & $\mathrm{S}$ & 3 & T. C. Wang \\
\hline 13640 & Asia & Taiwan & Solanaceae & $\mathrm{A} 1$ & $\mathrm{~S}$ & $\mathrm{AD}$ & T. C. Wang \\
\hline 13641 & Asia & Taiwan & Solanaceae & A1 & $\mathrm{S}$ & 3 & T. C. Wang \\
\hline 13642 & Asia & Taiwan & Solanaceae & $\mathrm{A} 2$ & $\mathrm{~S}$ & 3 & T. C. Wang \\
\hline 13644 & N. America & US, CA & Solanaceae & A2 & $\mathrm{S}$ & 9 & J. P. Prince \\
\hline 13645 & N. America & US, CA & Solanaceae & $\mathrm{A} 2$ & $\mathrm{~S}$ & 9 & J. P. Prince \\
\hline 13647 & N. America & US, CA & Solanaceae & A2 & $S$ & 5 & J. P. Prince \\
\hline 13649 & N. America & US, CA & Solanaceae & $\mathrm{A} 1$ & $\mathrm{~S}$ & 6 & J. P. Prince \\
\hline 13652 & N. America & US, CA & Solanaceae & A2 & $\mathrm{S}$ & 9 & N. Kabir \\
\hline 13653 & N. America & US, NM & Solanaceae & A1 & $\mathrm{S}$ & $\mathrm{AD}$ & N. Kabir \\
\hline 13654 & N. America & Mexico & Solanaceae & $\mathrm{A} 2$ & $\mathrm{~S}$ & 4 & N. Kabir \\
\hline 13655 & N. America & US, FL & Solanaceae & A2 & IS & 6 & N. Kabir \\
\hline 13656 & N. America & US, NM & Solanaceae & $\mathrm{A} 2$ & $\mathrm{~S}$ & 4 & N. Kabir \\
\hline 13657 & N. America & Mexico & Solanaceae & A2 & $\mathrm{S}$ & 8 & N. Kabir \\
\hline 13658 & Europe & Italy & Solanaceae & A1 & S & 6 & N. Kabir \\
\hline 13659 & N. America & Mexico & Solanaceae & $\mathrm{A} 2$ & $\mathrm{~S}$ & $\mathrm{AD}$ & N. Kabir \\
\hline 13660 & Asia & Korea & Solanaceae & A1 & $S$ & 8 & N. Kabir \\
\hline 13662 & Asia & Thailand & Solanaceae & $\mathrm{A} 1$ & $\mathrm{~S}$ & 3 & N. Kabir \\
\hline 13663 & N. America & US, FL & Solanaceae & A1 & $\mathrm{S}$ & 6 & N. Kabir \\
\hline 13665 & Asia & Taiwan & Solanaceae & $\mathrm{A} 2$ & S & 3 & P. J. Ann \\
\hline 13666 & Asia & Taiwan & Solanaceae & $\mathrm{A} 1$ & $\mathrm{~S}$ & 3 & P. J. Ann \\
\hline 13667 & Asia & Taiwan & Solanaceae & A2 & $\mathrm{S}$ & 3 & P. J. Ann \\
\hline 13668 & Asia & Taiwan & Solanaceae & $\mathrm{A} 1$ & $\mathrm{~S}$ & 3 & P. J. Ann \\
\hline 13669 & Asia & Taiwan & Solanaceae & A2 & $\mathrm{S}$ & 3 & P. J. Ann \\
\hline 13670 & Asia & Taiwan & Piperaceae & $\mathrm{A} 1$ & I & $\mathrm{AD}$ & P. J. Ann \\
\hline 13671 & Asia & Taiwan & Piperaceae & A2 & IS & 3 & P. J. Ann \\
\hline 13672 & Asia & Taiwan & Cucurbitaceae & $\mathrm{A} 1$ & $\mathrm{~S}$ & 3 & P. J. Ann \\
\hline 13673 & Asia & Taiwan & Cucurbitaceae & A2 & $\mathrm{S}$ & 3 & P. J. Ann \\
\hline 13674 & N. America & US, OH & Cucurbitaceae & A1 & A & 7 & S. Miller \\
\hline 13675 & N. America & US, OH & Solanaceae & A2 & $\mathrm{S}$ & 5 & S. Miller \\
\hline 13677 & N. America & US, OH & Cucurbitaceae & A2 & $\mathrm{S}$ & 3 & S. Miller \\
\hline 13678 & N. America & $\mathrm{US}, \mathrm{OH}$ & Cucurbitaceae & $\mathrm{A} 2$ & $\mathrm{~S}$ & 5 & S. Miller \\
\hline 13692 & N. America & US, NM & Solanaceae & A1 & $\mathrm{S}$ & 3 & ATCC \\
\hline 13693 & S. America & Uruguay & Solanaceae & A2 & $\mathrm{S}$ & 4 & R. Bernal \\
\hline 13694 & S. America & Uruguay & Solanaceae & A2 & $\mathrm{S}$ & 4 & R. Bernal \\
\hline 13695 & S. America & Uruguay & Solanaceae & A2 & $\mathrm{S}$ & 4 & R. Bernal \\
\hline 13699 & Asia & Japan & Solanaceae & A2 & $\mathrm{S}$ & 4 & S. Uemtsu \\
\hline 13700 & Asia & Japan & Solanaceae & A2 & $\mathrm{S}$ & 4 & S. Uemtsu \\
\hline 13704 & N. America & US, NJ & Fabaceae & $\mathrm{A} 2$ & $\mathrm{~S}$ & 4 & N. Gregory \\
\hline 13705 & N. America & US, NJ & Cucurbitaceae & A2 & $\mathrm{S}$ & 4 & N. Gregory \\
\hline 13706 & N. America & US, NJ & Cucurbitaceae & A2 & IS & 5 & N. Gregory \\
\hline 13707 & N. America & US, NJ & Fabaceae & A2 & IS & 5 & N. Gregory \\
\hline 13708 & N. America & US, NJ & Cucurbitaceae & A2 & IS & 5 & N. Gregory \\
\hline 13709 & N. America & US, NJ & Fabaceae & $\mathrm{A} 2$ & $\mathrm{~S}$ & 4 & N. Gregory \\
\hline 13710 & N. America & US, NJ & Fabaceae & A2 & $\mathrm{S}$ & 8 & N. Gregory \\
\hline 13711 & N. America & US, NJ & Fabaceae & $\mathrm{A} 1$ & $\mathrm{~S}$ & 8 & N. Gregory \\
\hline 13712 & S. America & Peru & Solanaceae & A2 & $\mathrm{S}$ & 2 & K. H. Lamour \\
\hline 13713 & S. America & Peru & Solanaceae & A2 & $\mathrm{S}$ & 2 & K. H. Lamour \\
\hline 13714 & S. America & Peru & Solanaceae & A2 & $\mathrm{S}$ & 2 & K. H. Lamour \\
\hline
\end{tabular}


length, LB ratio, pedicle length, sporangial density, and growth diameter at 32,35 , and $38^{\circ} \mathrm{C}$. A Box-Cox transformation (PROC TRANSREG) was used for sporangial width. The sporangial length, pedicle length, LB ratio, and growth diameter were log transformed and the sporangial density was square root transformed prior to analyses to satisfy the assumption of normality.

$P$. tropicalis and isolates with an intermediate genotype were removed from the dataset prior to further statistical analysis, leaving only P. capsici sensu stricto isolates. Data were subjected to analysis of variance, and Tukey's HSD was used for separation of means to account for unequal sample sizes between subgroups in a category $(P=0.05)$. The contrast option in PROC MIXED was used to compare isolates from vegetable crops and nonvegetable hosts. Isolates were arbitrarily grouped by predominant genetic cluster, continent of origin, mefenoxam sensitivity, mating type, and host family of origin; these were the main factors in ANOVA analyses. Isolate was considered a random factor. The response variables were sporangial width, sporangial length, LB ratio, pedicle length, oospore diameter, and sporangial density. A Box-Cox transformation (PROC TRANSREG) was used for sporangial width. The sporangial length, pedicle length, and LB ratio were log transformed, and the sporangial density was square root transformed prior to analyses to satisfy the assumption of normality.

\section{Results}

$P$. capsici sporangia were caducous and papillate with long pedicles ranging in length from 24 to $177 \mu$ m (mean $68 \pm 41 \mu \mathrm{m}$ ). Sporangia for isolates determined to be $P$. tropicalis or intermediate isolates were also caducous and papillate with long pedicles ranging from 20 to $260 \mu \mathrm{m}$ (mean $92 \pm 44 \mu \mathrm{m}$ ) and 20 to $152 \mu \mathrm{m}$ (mean $67 \pm 33 \mu \mathrm{m}$ ), respectively. Overall, the mean pedicle length was significantly larger for $P$. tropicalis than for $P$. capsici, with intermediate isolates showing an intermediate mean pedicle length (Table 2). The mean sporangial length and width for each $P$. capsici isolate ranged from 38.0 to $59.5 \mu \mathrm{m}$ long and 22.5 to $34.5 \mu \mathrm{m}$ wide. The overall mean length for all $P$. capsici isolates was $47.1 \pm$ $8.4 \mu \mathrm{m}$ long, and the overall mean width was $28.1 \pm 4.4 \mu \mathrm{m}$. The LB ratio of all of the $P$. capsici isolates was between 1.3 and 2.1, with a mean ratio of $1.7 \pm 0.3$ (Table 2). Twenty-six $P$. capsici isolates had $\mathrm{LB}$ ratios $>1.8$, and these isolates belonged to all nine genetic clusters (Fig. 1A).

P. tropicalis isolates presented a mean length and width of 39.6 to $49.0 \mu \mathrm{m}$ long and 21.7 to $26.3 \mu \mathrm{m}$ wide, and intermediate isolates ranged from 32.8 to $50.1 \mu \mathrm{m}$ long and 18.2 to $24.6 \mu \mathrm{m}$ wide. The overall mean length and width for all $P$. tropicalis isolates was $44.0 \pm 7.3 \mu \mathrm{m}$ long and $23.8 \pm 3.4 \mu \mathrm{m}$ wide. Intermediate isolates presented an overall mean length and width of $41.4 \pm 9.8 \mu \mathrm{m}$ long and $20.7 \pm 3.5 \mu \mathrm{m}$ wide. The $\mathrm{LB}$ ratio of all of the $P$. tropicalis isolates was 1.7 to 2.2 , with a mean of $1.9 \pm 0.3$. The LB ratio of the intermediate isolates was 1.7 to 2.1 , with an overall mean of $2.0 \pm 0.3$ (Table 2). Overall, $P$. capsici had a larger mean spo- rangial width and smaller LB ratio than $P$. tropicalis or intermediate isolates. Intermediate isolates had a smaller mean sporangial width than $P$. capsici or $P$. tropicalis. $P$. tropicalis and intermediate isolates had a statistically similar LB ratio. $P$. capsici had the largest mean sporangial length, which was similar to the mean length for $P$. tropicalis isolates. Intermediate isolates had shorter sporangia than P. capsici, but these were similar to P. tropicalis (Table 2).

When $P$. capsici isolates were grouped by predominant genetic cluster, no differences were observed in LB ratio $(P=0.1965)$ or pedicle length $(P=0.6148)$, but differences were observed in the mean sporangial width $(P=0.0001)$ and length $(P=0.0387)$ of isolates (Table 3 ). Isolates belonging predominantly to clusters four and six presented the smallest and largest sporangia, respectively. The mean sporangial length of isolates in the other genetic clusters was not significantly different than the mean sporangial length for clusters four and six. Clusters three, six, seven, and eight had the greatest mean sporangial width. Isolates belonging to clusters one, two, five, and nine had sporangia that were not significantly different in width from isolates belonging to other clusters (Table 3).

When isolates were grouped by host family of origin, differences were observed in LB ratio $(P=0.0031)$ and pedicle length $(P$ $=0.0017)$, but not in sporangial width $(P=0.6580)$ and length $(P=$ 0.1552 ) (Table 4). Isolates from solanaceous and cucurbitaceous hosts had the largest and smallest mean LB ratio, respectively. Isolates from hosts belonging to the Piperaceae, Proteaceae, Sterculiaceae, and Fabaceae did not significantly differ in LB ratio from isolates from other host families. Isolates from vegetable hosts belonging to the Solanaceae, Fabaceae, and Cucurbitaceae had shorter pedicles than isolates from piperaceous hosts. Isolates from hosts belonging to the Proteaceae and Sterculiaceae had mean pedicle lengths that were not significantly different than isolates from other host families.

Differences in LB ratio $(P=0.0002)$, pedicle length $(P=$ $0.0146)$, and sporangial length $(P<0.0001)$ were apparent when $P$. capsici isolates were grouped by continent. Isolates from Asia had longer sporangia, longer pedicles, and larger LB ratios than isolates from North America. In addition, North American isolates had shorter sporangia than isolates from Europe and a smaller LB ratio than isolates from South America. Isolates from Asia and Europe had longer sporangia than isolates from North America. Isolates from Asia and South America had larger LB ratios than isolates from North America. Isolates from Asia also had longer pedicles than isolates from North America. Differences in sporangial width were less clear $(P=0.0341)$, with statistically similar means for each continent using the more conservative Tukey's HSD method (Table 5).

When isolates were grouped by mating type and sensitivity to mefenoxam, no differences were observed for sporangial width $(P$ $>0.0664)$ and length $(P>0.0993)$, or for pedicle length $(P>$ $0.6126)$. LB ratios were not different for isolates grouped by sensitivity to mefenoxam $(P=0.4140)$, but the $\mathrm{LB}$ ratio was statistically

Table 2. Characteristics of Phytophthora isolates included in this study

\begin{tabular}{|c|c|c|c|c|}
\hline Phytophthora spp. & P. capsici & Intermediate $^{x}$ & P. tropicalis & $P$ value \\
\hline $\mathrm{n}^{\mathrm{y}}$ & 124 & 5 & 9 & \\
\hline Pedicle length $(\mu \mathrm{m})^{\mathrm{z}}$ & $67.9 \pm 41.5 \mathrm{~b}$ & $67.2 \pm 32.9 \mathrm{ab}$ & $92.2 \pm 44.3 \mathrm{a}$ & 0.0066 \\
\hline Sporangial length $(\mu \mathrm{m})^{\mathrm{z}}$ & $47.1 \pm 8.4 \mathrm{a}$ & $41.4 \pm 9.8 \mathrm{~b}$ & $44.0 \pm 7.3 \mathrm{ab}$ & 0.0027 \\
\hline Sporangial width $(\mu \mathrm{m})^{\mathrm{z}}$ & $28.1 \pm 4.4 \mathrm{a}$ & $20.7 \pm 3.5 \mathrm{c}$ & $23.8 \pm 3.4 \mathrm{~b}$ & $<0.0001$ \\
\hline Length:breadth ratio ${ }^{z}$ & $1.7 \pm 0.3 \mathrm{~b}$ & $2.0 \pm 0.3 \mathrm{a}$ & $1.9 \pm 0.3 \mathrm{a}$ & $<0.0001$ \\
\hline Chlamydospores (n) & 35 & 1 & 2 & \\
\hline Growth at $32^{\circ} \mathrm{C}(\mathrm{n})$ & 124 & 5 & 9 & \\
\hline Growth at $32^{\circ} \mathrm{C}(\mathrm{cm})^{\mathrm{z}}$ & $4.2 \pm 0.6 \mathrm{a}$ & $2.1 \pm 1.7 \mathrm{c}$ & $3.1 \pm 1.6 \mathrm{~b}$ & $<0.0001$ \\
\hline Growth at $35^{\circ} \mathrm{C}(\mathrm{n})$ & 122 & 2 & 8 & \\
\hline Growth at $35^{\circ} \mathrm{C}(\mathrm{cm})^{\mathrm{z}}$ & $1.7 \pm 2.5 \mathrm{a}$ & $0.2 \pm 0.4 \mathrm{~b}$ & $0.3 \pm 0.2 b$ & $<0.0001$ \\
\hline Growth at $38^{\circ} \mathrm{C}(\mathrm{n})$ & 0 & 0 & 0 & \\
\hline Growth at $38^{\circ} \mathrm{C}(\mathrm{cm})^{\mathrm{z}}$ & $0.0 \pm 0.1 \mathrm{a}$ & $0.0 \pm 0.0 \mathrm{a}$ & $0.0 \pm 0.0 \mathrm{a}$ & 0.1493 \\
\hline Sporangial density $\left(\text { sporangia/ } / \mathrm{cm}^{2}\right)^{z}$ & $515,151 \pm 10,717 \mathrm{ab}$ & $980,720 \pm 88,599 \mathrm{a}$ & $417,638 \pm 48,832 b$ & 0.0200 \\
\hline
\end{tabular}

\footnotetext{
${ }^{\mathrm{x}}$ Isolates that were identified as $P$. capsici in some genes and P. tropicalis in others.

${ }^{\mathrm{y}} \mathrm{n}=$ number of isolates.

${ }^{z}$ Mean \pm standard deviation. Means that share letters within a row are not significantly different (Tukey's HSD, $P<0.05$ ).
} 
greater $(P=0.0269)$ for $\mathrm{A} 2$ isolates $(1.72 \pm 0.01)$ than for A1 isolates $(1.66 \pm 0.01)$.

While no significant differences were observed for sporangial density on culture medium when $P$. capsici isolates were grouped by genetic cluster $(P=0.3204)$, mating type $(P=0.0599)$, sensitivity to mefenoxam $(P=0.4862)$, or host family $(P=0.3577)$, differences were observed for isolates when grouped by continent $(P=$ 0.0033, Table 5). Isolates collected in North America produced more sporangia in culture than isolates from South America. Isolates from Asia, Europe, and Africa produced a statistically similar number of sporangia as isolates from other continents (Table 5). While only four $P$. capsici isolates from nonvegetable hosts were included in this study, these isolates generally produced fewer sporangia than isolates from vegetable crops $(P=0.0253$, data not shown). The five intermediate isolates included in this study produced more sporangia on culture media than $P$. tropicalis isolates. Mean sporangial production by $P$. capsici on culture media was not significantly different than production by $P$. tropicalis or isolates with an intermediate genotype (Table 2).
All of the $P$. capsici isolates produced oospores when paired with known A1 or A2 mating types on UCV8. The average $P$. capsici oospore diameter was $28.5 \pm 3.4 \mu \mathrm{m}$, and oospores ranged in diameter from 21.7 to $36.9 \mu \mathrm{m}$. No differences were noted in $P$. capsici oospore diameter when the isolates were grouped by genetic cluster $(P=0.5650)$, host family of origin $(P=0.3324)$, mating type $(P=0.4655)$, mefenoxam sensitivity $(P=0.0655)$, or by continent $(P=0.0686)$ (data not shown).

Chlamydospores were produced by 35 isolates from hosts in the Cucurbitaceae (14 of 46), Fabaceae (2 of 12), and Solanaceae (19 of 63). No chlamydospores were produced by the four isolates of $P$. capsici retrieved from nonvegetable hosts (Piperaceae, Proteaceae, and Sterculiaceae) (data not shown). Isolates that produced chlamydospores belonged to all of the clusters except one and two (Fig. 1B). Isolates belonging to cluster three seemed to have a greater probability of producing chlamydospores than isolates belonging to other clusters (Fig. 1B); 14 of 35 isolates that produced chlamydospores belonged predominantly to cluster three. Chlamydospores were produced

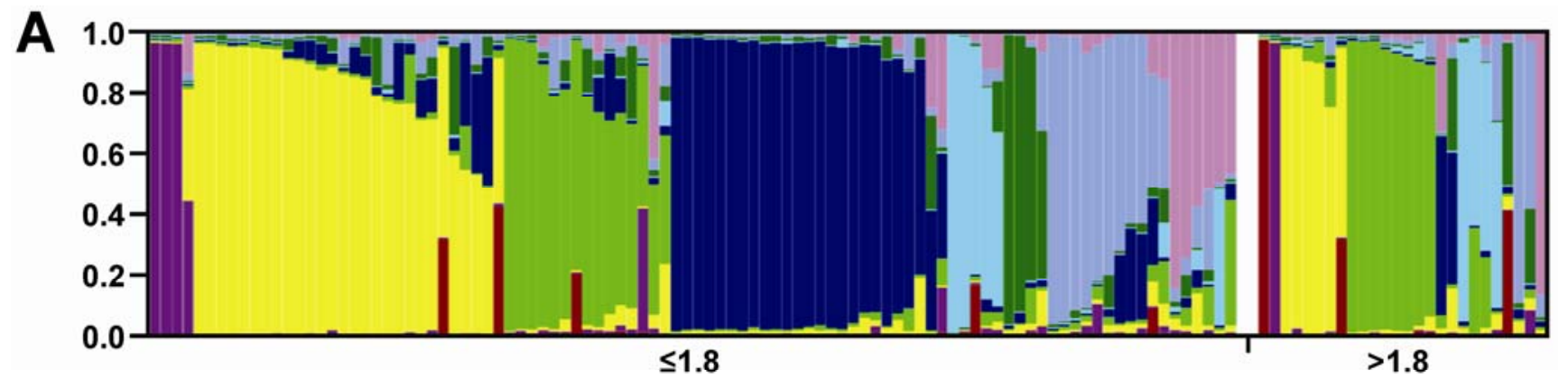

Length:breadth ratio

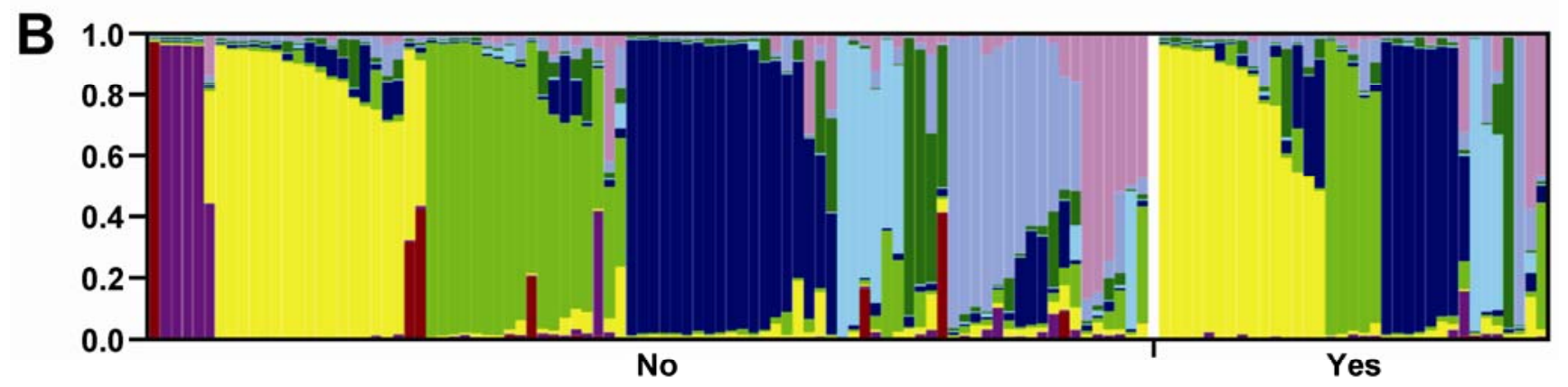

\section{Chlamydospore Production}

Fig. 1. Genetic structure of Phytophthora capsici isolates grouped by $\mathbf{A}$, length:breadth ratio, and $\mathbf{B}$, chlamydospore production. Each bar represents a single $P$. capsici isolate, which may be partitioned into colored segments representing an individual's proportionate genetic membership in a given Kth genetic cluster. Colors correspond to clusters as follows: dark red, one; purple, two; yellow, three; light green, four; dark blue, five; aqua, six; dark green, seven; light blue, eight; and pink, nine.

Table 3. Differences in morphological characteristics of Phytophthora capsici isolates grouped by predominant genetic cluster membership

\begin{tabular}{lrccccc}
\hline $\mathbf{G C}^{\mathbf{w}}$ & $\mathbf{n}^{\mathbf{x}}$ & ${\text { Sporangial length }(\boldsymbol{\mu m})^{\mathbf{y}}}$ & ${\text { Sporangial width }(\boldsymbol{\mu m})^{\mathbf{y}}}$ & LB ratio $^{\mathbf{y}}$ & ${\text { Pedicle length }(\boldsymbol{\mu m})^{\mathbf{y}}}^{\text {Sporangial density }^{\mathbf{y}, \mathbf{z}}}$ \\
\hline 1 & 1 & $45.4 \pm 9.8 \mathrm{ab}$ & $25.0 \pm 3.1 \mathrm{ab}$ & $1.8 \pm 0.3$ & $76.0 \pm 39.9$ & $144,792 \pm 21,161$ \\
2 & 4 & $48.9 \pm 7.6 \mathrm{ab}$ & $27.9 \pm 3.7 \mathrm{ab}$ & $1.8 \pm 0.3$ & $56.4 \pm 24.3$ & $393,490 \pm 74,862$ \\
3 & 33 & $47.4 \pm 8.0 \mathrm{ab}$ & $28.8 \pm 4.4 \mathrm{a}$ & $1.7 \pm 0.3$ & $70.5 \pm 39.3$ & $477,424 \pm 17,332$ \\
4 & 20 & $44.5 \pm 8.5 \mathrm{~b}$ & $25.9 \pm 4.4 \mathrm{~b}$ & $1.7 \pm 0.3$ & $63.1 \pm 35.8$ & $600,844 \pm 28,329$ \\
5 & 25 & $46.4 \pm 8.4 \mathrm{ab}$ & $27.5 \pm 4.0 \mathrm{ab}$ & $1.7 \pm 0.2$ & $64.7 \pm 37.9$ & $542,742 \pm 22,217$ \\
6 & 9 & $51.6 \pm 10.0 \mathrm{a}$ & $29.3 \pm 3.1 \mathrm{a}$ & $1.8 \pm 0.3$ & $75.4 \pm 38.3$ & $387,060 \pm 36,153$ \\
7 & 4 & $46.5 \pm 7.7 \mathrm{ab}$ & $30.0 \pm 5.0 \mathrm{a}$ & $1.6 \pm 0.2$ & $64.4 \pm 44.1$ & $467,031 \pm 53,939$ \\
8 & 13 & $48.1 \pm 7.2 \mathrm{ab}$ & $29.3 \pm 3.9 \mathrm{a}$ & $1.7 \pm 0.2$ & $66.5 \pm 35.8$ & $553,157 \pm 34,796$ \\
9 & 5 & $47.7 \pm 7.4 \mathrm{ab}$ & $27.6 \pm 4.0 \mathrm{ab}$ & $1.7 \pm 0.2$ & $82.7 \pm 49.9$ & $544,500 \pm 50,666$ \\
\hline
\end{tabular}

${ }^{\mathrm{w}} \mathrm{GC}=$ genetic cluster. Isolates $(\mathrm{n}=10)$ that were highly admixed were excluded from these analyses.

${ }^{\mathrm{x}} \mathrm{n}=$ number of isolates.

${ }^{y}$ Mean \pm standard deviation. Means with no letter or that share letters within a column are not significantly different (Tukey's HSD, $P<0.05$ ).

${ }^{z}$ Sporangia $/ \mathrm{cm}^{2}$. 
by one of five isolates with an intermediate genotype and two of nine $P$. tropicalis isolates (Table 2).

All of the P. capsici, P. tropicalis, and intermediate isolates grew well at $32^{\circ} \mathrm{C}$. At $35^{\circ} \mathrm{C}$, two of 124 P. capsici $(13634,13693)$, one of nine P. tropicalis (13607), and three of five intermediate (13366, 13367,13368 ) isolates were unable to grow (Table 2). When cultures were incubated at 32 or $35^{\circ} \mathrm{C}$, the mean growth diameter of $P$. capsici was greater than the mean growth diameter of $P$. tropicalis and isolates with an intermediate genotype. When isolates were incubated at $38^{\circ} \mathrm{C}$ for 5 days, all of the isolates grew poorly $(<0.5$ $\mathrm{cm})$, and there were no significant differences in mean growth diameter among species (Table 2).

\section{Discussion}

The $P$. capsici isolates in this study showed significant variation in morphological and physiological characteristics. The mean sporangial length and width for each $P$. capsici isolate ranged from 38 to $60 \mu \mathrm{m}$ long and 23 to $35 \mu \mathrm{m}$ wide, in agreement with other studies that reported sporangia to range from 33 to $66 \mu \mathrm{m}$ long and 17 to $43 \mu \mathrm{m}$ wide $(3,17,22,28,36)$. Ristaino (28) noted that $P$. capsici isolates from cucurbit hosts (56 to $63 \mu \mathrm{m}$ long) generally produced longer sporangia than isolates collected from pepper (39 to $60 \mu \mathrm{m}$ long). In our study, differences in sporangial length and width were not apparent when isolates were grouped by host family of origin, in contrast to what was previously observed by Ristaino. The range of sporangial lengths in our study were 39 to $53 \mu \mathrm{m}$ for isolates from cucurbit hosts and 38 to $59 \mu \mathrm{m}$ for isolates from solanaceous hosts (38 to $59 \mu \mathrm{m}$ for isolates from pepper). Islam et al (17) found lengths of 42 to $55 \mu \mathrm{m}$ for isolates from processing pumpkin, which is more similar to lengths observed in our study than to those reported by Ristaino for cucurbit isolates (28). Significant differences in the mean sporangial width and length of $P$. capsici isolates were noted when isolates were grouped by genetic cluster or geography, but not when isolates were grouped by mating type and sensitivity to mefenoxam. $P$. tropicalis isolates presented a mean sporangial length and width of 39.6 to $49.0 \mu \mathrm{m}$ long and 21.7 to $26.3 \mu \mathrm{m}$ wide, and intermediate isolates had sporangia that ranged from 32.8 to $50.1 \mu \mathrm{m}$ long and 18.2 to $24.6 \mu \mathrm{m}$ wide in our study. Mchau and Coffey (22) reported sporangial lengths and widths of 33 to 66 and 19 to $32 \mu \mathrm{m}$, respectively, for $P$. capsici CAPB isolates, which likely correspond to $P$. tropicalis.

The LB ratio of all of the $P$. capsici isolates in this study ranged from 1.3 to 2.1. Mchau and Coffey (22) found a similar range of LB ratios, with isolates belonging to CAPA (likely $P$. capsici sensu stricto) having ratios of 1.3 to 2.0 and isolates belonging to CAPB, which likely represents $P$. tropicalis isolates, having ratios of 1.4 to 2.1. Other previous studies reported LB ratios of 1.4 to 1.8 for isolates from pepper $(28,36)$ and 1.3 to 1.8 for isolates from cucurbits $(17,28)$. We found LB ratios ranging from 1.4 to 2.1 for isolates from solanaceous hosts and 1.3 to 1.8 for isolates from cucurbitaceous hosts, which generally agrees with previous findings. Differences in mean LB ratios were apparent when $P$. capsici isolates were grouped by host family of origin, mating type, and continent of origin, but not when isolates were grouped by predominant genetic cluster or mefenoxam sensitivity. Aragaki and Uchida (3) suggest that generally isolates belonging to $P$. capsici should have an LB ratio $<1.8$, and isolates belonging to $P$. tropicalis should have an LB ratio $>1.8$. In our study, $21 \%$ of $P$. capsici isolates (26 of 124), 67\% of $P$. tropicalis (6 of 9), and $80 \%$ of intermediate isolates ( 4 of 5 ) had LB ratios $>1.8$, and would be identified as $P$. tropicalis if this one morphological feature was used exclusively. Most of the $P$. capsici isolates with an LB ratio $>1.8$ were originally isolated from solanaceous hosts (23 of 26). Clearly, LB ratio is an important morphological trait for separation of $P$. capsici from $P$. tropicalis; however, since some $P$. capsici and most intermediate isolates possessed LB ratios $>1.8$, separation of $P$. capsici and intermediate isolates from $P$. tropicalis requires a more thorough examination of isolates, and molecular methods using multiple genes are likely necessary.

Pedicle length has been noted to be a highly variable feature in previous studies $(17,22,28)$, and pedicle lengths also varied widely in our study. Pedicle lengths ranged from 24 to $177 \mu$ m (mean $68 \pm$ $41 \mu \mathrm{m}$ ) for $P$. capsici, from 20 to $260 \mu \mathrm{m}$ (mean $92 \pm 44 \mu \mathrm{m}$ ) for $P$. tropicalis, and from 24 to $177 \mu \mathrm{m}$ (mean $67 \pm 33 \mu \mathrm{m}$ ) for intermediate isolates. Mean pedicle lengths were 65 and $87 \mu \mathrm{m}$ for $P$. capsici and $P$. tropicalis, respectively, in a previous study by Aragaki and Uchida (3). Pedicle lengths for $P$. capsici isolates ranged from 32 to $123 \mu \mathrm{m}$ in previous studies $(17,22,28)$, which is a slightly more limited range than was observed in our study. When isolates were grouped by host family or continent of origin, significant differences were observed in pedicle lengths, but differences were not apparent when isolates were grouped by other categories.

When cultures were incubated in liquid media, 35 of 124 (28\%) $P$. capsici isolates and two of nine (22\%) P. tropicalis isolates produced chlamydospores. In Aragaki and Uchida's study (3), no $P$. capsici isolates and most (46 of 53, 87\%) P. tropicalis isolates produced chlamydospores. Similarly, no isolates from temperate hosts, but the majority ( 8 of 10) of isolates from nonvegetable

Table 4. Differences in morphological characteristics of Phytophthora capsici isolates grouped by host family of origin

\begin{tabular}{lcccccc}
\hline Host $^{\mathbf{w}}$ & $\mathbf{n}^{\mathbf{x}}$ & ${\text { Sporangial length }(\boldsymbol{\mu m})^{\mathbf{y}}}$ & ${\text { Sporangial width }(\boldsymbol{\mu m})^{\mathbf{y}}}$ & LB ratio $^{\mathbf{y}}$ & ${\text { Pedicle length }(\boldsymbol{\mu m}){ }^{\mathbf{y}}}^{\text {Sporangial density }^{\mathbf{y}, \mathbf{z}}}$ \\
\hline $\mathrm{Pi}$ & 3 & $48.6 \pm 5.5$ & $27.4 \pm 3.2$ & $1.8 \pm 0.2 \mathrm{ab}$ & $144.6 \pm 110.6 \mathrm{a}$ & $320,577 \pm 41,642$ \\
$\mathrm{Pr}$ & 1 & $48.9 \pm 8.5$ & $30.9 \pm 3.1$ & $1.6 \pm 0.2 \mathrm{ab}$ & $99.9 \pm 38.3 \mathrm{ab}$ & $93,750 \pm 27,594$ \\
$\mathrm{St}$ & 1 & $45.4 \pm 9.8$ & $25.0 \pm 3.1$ & $1.8 \pm 0.3 \mathrm{ab}$ & $76.0 \pm 39.9 \mathrm{ab}$ & $144,792 \pm 21,161$ \\
$\mathrm{So}$ & 63 & $48.5 \pm 8.8$ & $28.1 \pm 4.4$ & $1.7 \pm 0.3 \mathrm{a}$ & $69.9 \pm 38.3 \mathrm{~b}$ & $533,760 \pm 15,072$ \\
$\mathrm{Fa}$ & 12 & $46.7 \pm 7.3$ & $28.4 \pm 5.1$ & $1.7 \pm 0.3 \mathrm{ab}$ & $59.7 \pm 30.0 \mathrm{~b}$ & $529,236 \pm 27,694$ \\
$\mathrm{Cu}$ & 44 & $45.6 \pm 7.8$ & $28.3 \pm 4.2$ & $1.6 \pm 0.2 \mathrm{~b}$ & $63.4 \pm 38.6 \mathrm{~b}$ & $512,296 \pm 18,820$ \\
\hline
\end{tabular}

${ }^{\mathrm{w}}$ Host family of origin. $\mathrm{Pi}=$ Piperaceae, $\mathrm{Pr}=$ Proteaceae, $\mathrm{St}=$ Sterculiaceae, $\mathrm{So}=$ Solanaceae, $\mathrm{Fa}=$ Fabaceae, $\mathrm{Cu}=\mathrm{Cucurbitaceae}$.

${ }^{\mathrm{x}} \mathrm{n}=$ number of isolates.

${ }^{y}$ Mean \pm standard deviation. Means with no letter or that share letters within a column are not significantly different (Tukey's HSD, $P<0.05$ ).

${ }^{\mathrm{z}}$ Sporangia/ $\mathrm{cm}^{2}$

Table 5. Differences in morphological characteristics of Phytophthora capsici isolates grouped by geography

\begin{tabular}{|c|c|c|c|c|c|c|}
\hline Continent & $\mathbf{n}^{\mathbf{x}}$ & Sporangial length $(\mu \mathrm{m})^{\mathrm{y}}$ & Sporangial width $(\mu \mathrm{m})^{y}$ & LB ratio $y$ & Pedicle length $(\mu \mathrm{m})^{\mathrm{y}}$ & Sporangial density ${ }^{y, z}$ \\
\hline N. America & 91 & $46.0 \pm 8.2 \mathrm{~b}$ & $28.0 \pm 4.3$ & $1.7 \pm 0.3 b$ & $64.6 \pm 37.1 \mathrm{~b}$ & $555,371 \pm 12,837 \mathrm{a}$ \\
\hline Asia & 18 & $50.5 \pm 7.6 \mathrm{a}$ & $28.8 \pm 4.6$ & $1.8 \pm 0.3 \mathrm{a}$ & $85.8 \pm 58.7 \mathrm{a}$ & $518,067 \pm 26,753 \mathrm{ab}$ \\
\hline Europe & 8 & $51.9 \pm 9.4 \mathrm{a}$ & $29.9 \pm 4.4$ & $1.7 \pm 0.2 \mathrm{ab}$ & $72.7 \pm 42.1 \mathrm{ab}$ & $318,646 \pm 29,040 a b$ \\
\hline S. America & 6 & $48.4 \pm 7.1 \mathrm{ab}$ & $26.1 \pm 3.7$ & $1.9 \pm 0.3 \mathrm{a}$ & $56.9 \pm 22.7 \mathrm{ab}$ & $220,139 \pm 25,856 \mathrm{~b}$ \\
\hline Africa & 1 & $45.4 \pm 9.8 \mathrm{ab}$ & $25.0 \pm 3.1$ & $1.8 \pm 0.3 \mathrm{ab}$ & $76.0 \pm 39.9 \mathrm{ab}$ & $144,792 \pm 21,161 \mathrm{ab}$ \\
\hline
\end{tabular}

${ }^{\mathrm{x}} \mathrm{n}=$ number of isolates.

${ }^{y}$ Mean \pm standard deviation. Means with no letter or that share letters within a column are not significantly different (Tukey's HSD, $P<0.05$ ).

${ }^{z}$ Sporangia $/ \mathrm{cm}^{2}$. 
hosts, some of which may have been $P$. tropicalis, formed chlamydospores in another study (4). Chlamydospores were not produced by isolates from solanaceous or cucurbitaceous hosts in the study by Ristaino (28), but were produced by $38 \%$ ( 21 of 57 ) of the isolates from pumpkin in the study by Islam et al. (17). Mchau and Coffey (22) found that 16 of 29 (55\%) CAP2 isolates produced chlamydospores, but none of the CAP1 isolates that likely represent $P$. capsici sensu stricto isolates produced chlamydospores. Hence, chlamydospore production also does not appear to be reliable for separation of $P$. tropicalis from $P$. capsici. The studies by Ristaino (28) and Islam et al. (17) incubated the cultures under similar conditions to the current study to check for chlamydospore production; in the study by Aragaki and Uchida (3), the isolates were incubated under warmer conditions, and this may account for some of the variation among studies. Curiously, previous studies have reported chlamydospore production by isolates from nonvegetable hosts $(1,32)$ and from pumpkin (17); in our study, chlamydospores were produced by isolates from hosts in the Cucurbitaceae, Fabaceae, and Solanaceae, and no chlamydospores were produced by the four isolates of $P$. capsici retrieved from nonvegetable hosts. However, $P$. tropicalis and intermediate isolates may have been included in some earlier studies $(1,32)$, which challenges comparisons between studies. In our study, cluster three contained a greater proportion of isolates that produced chlamydospores than other clusters. Isolates belonging predominantly to cluster three were collected from North America and Asia. Cluster three contains the type culture for $P$. capsici from New Mexico, which did not produce chlamydospores in our study.

Oospore diameters for P. capsici isolates ranged from 22 to 37 $\mu \mathrm{m}$ in our study, in agreement with previous studies $(17,22)$, and is a slightly larger range than was reported by Ristaino (28). Oospore diameters did not differ significantly when isolates were grouped by genetic cluster, host family of origin, mating type, mefenoxam sensitivity, or geography. Previous studies $(3,4,22)$ have suggested that growth at $35^{\circ} \mathrm{C}$ is a good character to separate $P$. capsici from $P$. tropicalis. A few isolates in these previous studies did not conform to this standard, but most did. In our study, most of the $P$. capsici isolates were able to grow at $35^{\circ} \mathrm{C}$ as in previous studies. Eight of the nine $P$. tropicalis isolates were also able to grow at $35^{\circ} \mathrm{C}$ in our study, which was unexpected. Overall, the growth diameter of $P$. capsici isolates at $35^{\circ} \mathrm{C}$ was significantly greater than the growth diameter of $P$. tropicalis isolates.

As sporangia and zoospores are responsible for polycyclic disease development (16), knowing the sporangial production potential of isolates may be useful to predict an isolate's ability to spread. Differences in sporangial production on culture medium were observed when isolates were grouped by geography (continent). Differences in sporangial production were not observed when isolates were grouped by genetic cluster, mating type, sensitivity to mefenoxam, or host family. A previous study by CaféFilho and Ristaino (6) found no significant differences in sporangial production on culture medium when a limited number of isolates were grouped by sensitivity to mefenoxam, in agreement with our results. The few isolates from nonvegetable hosts included in our study generally produced fewer sporangia than isolates from vegetable hosts; in a previous study looking at isolate virulence, a similar trend was observed, where isolates from nonvegetable hosts were generally less virulent on vegetable fruits than isolates from vegetable hosts (L. L. Granke, L. M. Quesada-Ocampo, and M. K. Hausbeck, unpublished). Sporangial density in culture medium was not correlated with isolate virulence (lesion diameter on cucumber, zucchini, tomato, and pepper fruits), but it was correlated with sporangial density (rated visually on a 0 to 2 scale, $0=$ no sporangia, $1=$ light sporulation, and $2=$ heavy sporulation) on infected fruits (data not shown, Pearson's correlation, $P=0.05$ ).

A wide range of variability was observed in morphological traits among $P$. capsici isolates in this study. When $P$. capsici isolates were compared to a limited number of $P$. tropicalis and intermediate isolates, clear phenotypic differences were not apparent for all isolates of each species. Hence, reliance on morphology alone could lead to misidentification of some isolates. In addition, previous studies have found differences in sporangial morphology of an oomycete pathogen between host species $(29,34)$ and by plant part on a single host (7), which further supports using molecular markers for identification.

\section{Acknowledgments}

We thank the many colleagues who provided isolates for this research. We thank all the members of the Hausbeck lab for their valuable suggestions and help. We especially thank A. Lebeis, R. Heslip, L. Henderson, and M. Wood for technical assistance. We thank J. J. Morrice for providing the program PST to generate genetic structure figures and for generating the maps used in Figure 1. We thank W. Yang for assistance with statistical analyses. This research was supported by the Michigan State University Michigan Agriculture Experiment Station Hatch Project 01966, the United States Department of Agriculture (USDA) Cooperative State Research, Education and Extension Service (CSREES), and the USDA-CSREES Special Research Grant under Awards No. 2006-34572-16902 and 2008-34572-16902.

\section{Literature Cited}

1. Alizadeh, A., and Tsao, P. H. 1985. Chlamydospore formation in Phytophthora palmivora MF4. Trans. Br. Mycol. Soc. 85:71-79.

2. Appiah, A. A., Flood, J., Archer, S. A., and Bridge, P. D. 2004. Molecular analysis of the major Phytophthora species on cacao. Plant Pathol. 53:209219.

3. Aragaki, M., and Uchida, J. Y. 2001. Morphological distinctions between Phytophthora capsici and P. tropicalis sp. nov. Mycologia 93:137-145.

4. Bowers, J. H., Martin, F. N., Tooley, P. W., and Luz, E. D. M. N. 2007. Genetic and morphological diversity of temperate and tropical isolates of Phytophthora capsici. Phytopathology 97:492-503.

5. Brasier, C. M. 1983. Problems and prospects in Phytophthora research. Pages 351-364 in: Phytophthora: Its Biology, Taxonomy, Ecology, and Pathology. D. C. Erwin, S. Bartnicki-Garcia, and P. H. Tsao, eds. American Phytopathological Society, St. Paul, MN.

6. Cafe-Filho, A. C., and Ristaino, J. B. 2008. Fitness of isolates of Phytophthora capsici resistant to mefenoxam from squash and pepper fields in North Carolina. Plant Dis. 92:1439-1443.

7. Delanoe, D. 1972. Biologie et Epidemiologie du mildiou du tournesol (in French). CETIOM Inform. Tech. 26:1-61.

8. Donahoo, R. S., and Lamour, K. H. 2008. Interspecific hybridization and apomixis between Phytophthora capsici and Phytophthora tropicalis. Mycologia 100:911-920.

9. Erwin, D. C., and Ribeiro, O. K. 1996. Phytophthora Diseases Worldwide. American Phytopathological Society, St. Paul, MN.

10. Forster, H., Oudemans, P., and Coffey, M. D. 1990. Mitochondrial and nuclear DNA diversity within six species of Phytophthora. Exp. Mycol 14:18-31.

11. Foster, J. M., and Hausbeck, M. K. 2010. Resistance of pepper to Phytophthora crown, root, and fruit rot is affected by isolate virulence. Plant Dis. 94:24-30.

12. Gevens, A. J., Ando, K., Lamour, K. H., Grumet, R., and Hausbeck, M. K. 2006. A detached cucumber fruit method to screen for resistance to Phytophthora capsici and effect of fruit age on susceptibility to infection. Plant Dis. 90:1276-1282.

13. Gevens, A. J., Donahoo, R. S., Lamour, K. H., and Hausbeck, M. K. 2007. Characterization of Phytophthora capsici from Michigan surface irrigation water. Phytopathology 97:421-428.

14. Granke, L. L., Quesada-Ocampo, L. M., and Hausbeck, M. K. 2010. Phenotypic diversity of Phytophthora capsici isolates from a worldwide collection. Pages 233-236 in: Cucurbitaceae 2010 Proceedings, American Society for Horticultural Science, Charleston SC.

15. Grote, D., and Claussen, W. 2001. Severity of root rot on tomato plants caused by Phytophthora nicotianae under nutrient- and light-stress conditions. Plant Pathol. 50:702-707.

16. Hausbeck, M. K., and Lamour, K. H. 2004. Phytophthora capsici on vegetable crops: Research progress and management challenges. Plant Dis. 88:1292-1303.

17. Islam, S. Z., Babadoost, M., Lambert, K. N., Ndeme, A., and Fouly, H. M. 2004. Characterization of Phytophthora capsici isolates from processing pumpkin in Illinois. Plant Dis. 89:191-197.

18. Lamour, K. H., and Hausbeck, M. K. 2000. Mefenoxam insensitivity and the sexual stage of Phytophthora capsici in Michigan cucurbit fields. Phytopathology 90:396-400.

19. Lamour, K. H., and Hausbeck, M. K. 2001. The dynamics of mefenoxam insensitivity in a recombining population of Phytophthora capsici characterized with amplified fragment length polymorphism markers. Phytopathology 91:553-557.

20. Lamour, K. H., and Hausbeck, M. K. 2003. Effect of crop rotation on the survival of Phytophthora capsici in Michigan. Plant Dis. 87:841-845.

21. Lee, B. K., Kim, B. S., Chang, S. W., and Hwang, B. K. 2001. Aggressiveness to pumpkin cultivars of isolates of Phytophthora capsici from pumpkin and pepper. Plant Dis. 85:497-500. 
22. Mchau, G. R. A., and Coffey, M. D. 1995. Evidence for the existence of two distinct subpopulations in Phytophthora capsici and a redescription of the species. Mycol. Res. 99:89-102.

23. Oudemans, P., and Coffey, M. D. 1991. A revised systematics of twelve papillate Phytophthora species based on isozyme analysis. Mycol. Res. 95:1025-1046.

24. Pritchard, J. K., Stephens, M., and Donnelly, P. 2000. Inference of population structure using multilocus genotypic data. Genetics 155:945-959.

25. Quesada-Ocampo, L. M., Fulbright, D. W., and Hausbeck, M. K. 2009. Susceptibility of Fraser fir to Phytophthora capsici. Plant Dis. 93:135-141.

26. Quesada-Ocampo, L. M., Granke, L. L., Mercier, M. R., Olsen, J., and Hausbeck, M. K. 2011. Investigating the genetic structure of Phytophthora capsici populations. Phytopathology 101:1061-1073.

27. Quesada-Ocampo, L. M., and Hausbeck, M. K. 2010. Resistance in tomato and wild relatives to crown and root rot caused by Phytophthora capsici. Phytopathology 100:619-627.

28. Ristaino, J. B. 1990. Intraspecific variation among isolates of Phytophthora capsici from pepper and cucurbit fields in North Carolina. Phytopathology 80:1253-1259.

29. Runge, F., and Thines, M. 2011. Host matrix has major impact on the mor- phology of Pseudoperonospora cubensis. Eur. J. Plant Pathol. 129:147-156.

30. Silvar, C., Duncan, J. M., Cooke, D. E. L., Williams, N. A., Diaz, J., and Merino, F. 2005. Development of specific PCR primers for identification and detection of Phytophthora capsici Leon. Eur. J. Plant Pathol. 112:43-52.

31. Tamietti, G., and Valentino, D. 2001. Physiological characterization of a population of Phytophthora capsici Leon. from northern Italy. J. Plant Pathol. 83:199-205.

32. Uchida, J. Y., and Aragaki, M. 1985. Occurrence of chlamydospores in Phytophthora capsici. Mycologia 77:832-835.

33. Waterhouse, G. M. 1963. Key to the Species of Phytophthora de Bary. Commonwealth Mycological Society, Kew, Surrey, UK.

34. Waterhouse, G. M., and Brothers, M. P. 1981. The taxonomy of Pseudoperonospora. Mycol. Pap. 148:1-28.

35. Zhang, Z. G., Zhang, J. Y., Zheng, X. B., Yang, Y. W., and Ko, W. H. 2004. Molecular distinctions between Phytophthora capsici and Ph. tropicalis based on ITS sequences of ribosomal DNA. J. Phytopathol. 152:358-364.

36. Zhijun, L. I., Weiping, L., Jinrong, Z., and Jianjun, L. 2007. Isolation and identification of Phytophthora capsici in Guangdong province and measurement of their pathogenicity and physiological race differentiation. Front. Agric. China 1:377-381. 